\title{
Pericarditis constrictiva crónica calcificada idiopática (concretio cordis)
}

\author{
Idiopathic calcific chronic constrictive pericarditis (concretio cordis)
}

\author{
Andrés Felipe Posada', Hernán Darío Aguirre ${ }^{2 凶}$, Juan José Duque ${ }^{3}$, Victor Gil Aldana4
}

Fecha correspondencia:

Recibido: diciembre 27 de 2015.

Revisado: junio 10 de 2016.

Aceptado: septiembre 11 de 2016.

Forma de citar:

Pericarditis constrictiva crónica calcificada idiopática (concretio cordis). Posada AF, Aguirre HD, Duque JJ, Gil Aldana V. Rev CES Med 2016; 30(2): 217-224.

Open access

(c) Copyright

Licencia creative commons

Ética de publicaciones

Revisión por pares

Gestión por Open Journal System

ISSN 0120-8705

e-ISSN 2215-9177

Sobre los autores:

1 Médico Internista, Centros especializados de San Vicente Fundación.

2 Médico Internista Clínica Universitaria Bolivariana- Hospital General de Medellín.

3 Médico Internista, Universidad de la Sabana, Chia, Colombia.

Comparte

\begin{abstract}
Resumen
La pericarditis constrictiva es una enfermedad poco frecuente que resulta de la pérdida de la elasticidad normal del saco pericárdico y la consiguiente cicatrización. Comprende diversas etiologías, pero en un gran porcentaje de los casos es diagnosticada como idiopática. Sus manifestaciones suelen ser crónicas, aunque sus variantes incluyen constricción subaguda, transitoria y oculta. Se presenta el caso de un paciente masculino de 53 años de edad con deterioro de su clase funcional asociado a disnea, frote pericárdico y hallazgos radiológicos de calcificación pericárdica, requiriendo pericardiectomía total.
\end{abstract}

Palabras clave: Pericarditis constrictiva, Concretio cordis, Pericardiectomía.

\section{Abstract}

Constrictive pericarditis is an uncommon condition resulting from loss of normal elasticity pericardial sac and consequent healing. Although a large percentage of cases are diagnosed as idiopathic. That pathology comprises various etiologies. Its manifestations are usually chronic, but variants include subacute and transient constriction. We present the case of a male patient of 53 years who presented functional class deterioration manifested by dyspnea, pericardial rub and radiological findings confirming pericardial calcification requiring total pericardiectomy.

Keywords: Constrictive pericarditis, Concretio cordis, Total pericardiectomy.

\section{Introducción}

El pericardio es un saco fibroelástico que recubre el corazón y contiene una capa fina de líquido. La pericarditis constrictiva es el resultado de la cicatrización y la consiguiente pérdida de la elasticidad normal del saco pericárdico. Las variantes de ésta pueden ser crónica, constricción subaguda, transitoria y oculta.

La pericarditis constrictiva puede ocurrir después de cualquier proceso de enfermedad pericárdica o bien como manifestación de enfermedad sistémica.

La presencia de calcificación pericárdica en una radiografía de tórax es muy sugerente de pericarditis constrictiva. Sin embargo, la mayoría de los pacientes con pericarditis constrictiva no tendrá calcificación pericárdica (1). 
Julio - Diciembre 2016 - Pág 218

4 Médico Internista, Cardiólogohemodinamista. Universidad de Antioquia.
La ecocardiografía es una herramienta diagnóstica que ayuda a diferenciar pericarditis constrictiva de cardiomiopatía restrictiva y debe ser la modalidad de imagen no invasiva inicial.
En 1982 Isner et al. demuestran el valor de la tomografía en el diagnóstico de pericarditis constrictiva, que actualmente es utilizada ampliamente para delinear la calcificación pericárdica y detectar complicaciones (2). Por su parte, la ecocardiografía es una herramienta diagnóstica que ayuda a diferenciar pericarditis constrictiva de cardiomiopatía restrictiva y debe ser la modalidad de imagen no invasiva inicial. La pericardiectomía es el tratamiento de elección en sitios altamente capacitados para dicho procedimiento.

A continuación se describe el caso de un paciente con pericarditis constrictiva crónica calcificada idiopática con manifestaciones clínicas de falla cardíaca aguda y a quien se le realizó pericardiectomía total exitosa, pese a tener indicadores de mal pronóstico y alto riesgo de mortalidad.

\section{Caso clínico}

Ingresa un hombre de 53 años con 10 días de deterioro de su clase funcional (III/IV de la NYHA) hasta presentar disnea con mínimos esfuerzos. Relataba dolor torácico de tipo pleurítico en hemitórax derecho, que se irradiaba al dorso, de intensidad severa y sin otra sintomatología adicional. No había presencia de ortopnea, ni disnea paroxística nocturna, tampoco edema de miembros inferiores y no había tenido pérdida de peso, ni fiebre.

Como antecedentes relevantes tuvo pericarditis hace cinco años con aparente resolución total. Fue diagnosticado como diabético hace cuatro años, actualmente en tratamiento con hipoglucemiantes orales con adecuado control metabólico. Fue fumador en su juventud con menos de cinco cigarrillos al día por aproximadamente 10 años.

Al examen físico presentaba ingurgitación yugular grado II, frote pericárdico, disminución de los ruidos respiratorios y matidez a la percusión en base del hemitórax derecho. Además, se le realizaron paraclínicos complementarios con imágenes que mostraban hallazgos típicos de pericarditis constrictiva calcificada (cuadro 1, figuras 1 y $\underline{2})$.

Cuadro 1. Paraclínicos de ingreso

\begin{tabular}{|c|c|c|c|}
\hline Prueba & Resultado & Prueba & Resultado \\
\hline Glicemia (mg/dl) & 84 & $\mathrm{BNP}(\mathrm{pg} / \mathrm{mL})$ & 91 \\
\hline ELISA anticuerpos antinucleares & Negativos $<1: 40$ & Troponina (ng/mL) & $<0,006$ \\
\hline $\begin{array}{l}\text { ELISA anticuerpos citoplasmáticos } \\
\text { neutrofilos }\end{array}$ & Negativos $<1: 16$ & Creatinina (mg/dl) & 0,7 \\
\hline Factor reumatoide por nefelometría (U/ml) & 3,1 & BUN (mg/dl) & 11 \\
\hline ELISA VIH & Negativo & Fibrinógeno & 258 \\
\hline PPD & Negativa & $\begin{array}{l}\text { Cultivo de pericardio para } \\
\text { micobacterias }\end{array}$ & Negativo \\
\hline
\end{tabular}




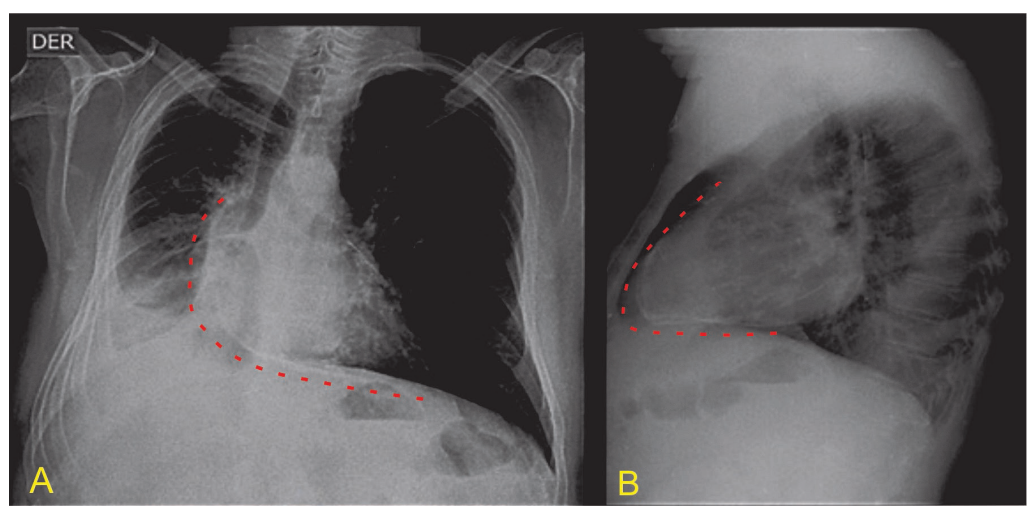

Figura 1. Radiografía de tórax. (A) Fontal (B) lateral. En ambas se observa clasificación pericárdica anular (concretio cordis)

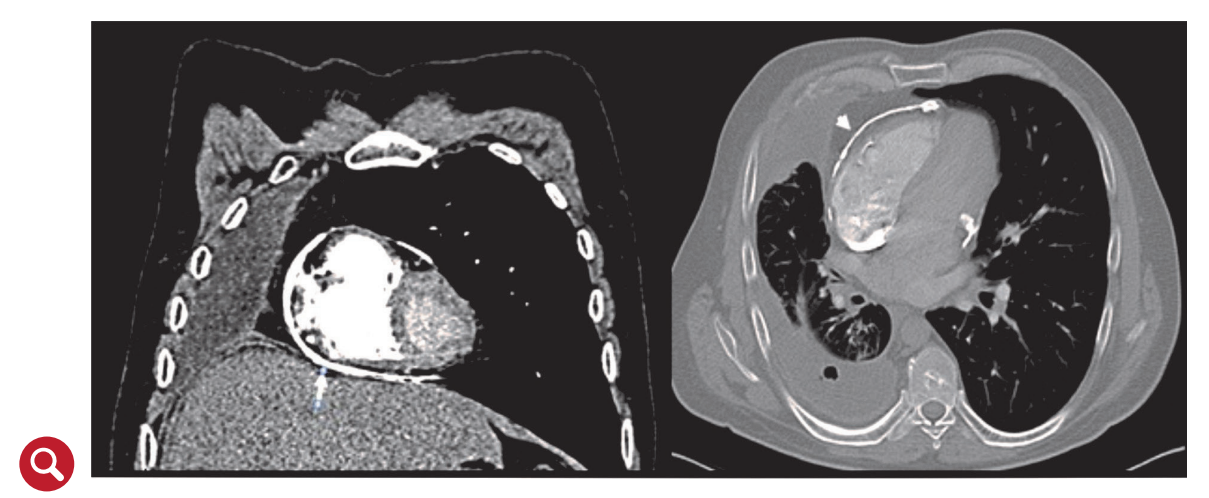

Figura 2. Tomografía de tórax. Se evidencia calcificación pericárdica anular y compromiso principalmente del ventrículo derecho (concretio cordis) y derrame pleural derecho

Debido a mala ventana acústica demostrada en la ecocardiografia transtorácica se realizó ecocardiografía transesofágica, en la que se evidenció movimiento anormal del septum interventricular con desplazamiento del ventrículo izquierdo en diástole, dilatación biauricular y engrosamiento difuso del pericardio, hallazgos que eran compatibles con pericarditis constrictiva, por lo que fue llevado a estudio hemodinámico prequirúrgico donde se evidenció incremento severo de la presión de la aurícula derecha; además, en la evaluación del ventrículo derecho, se observó el signo de raíz cuadrada (figura 3).

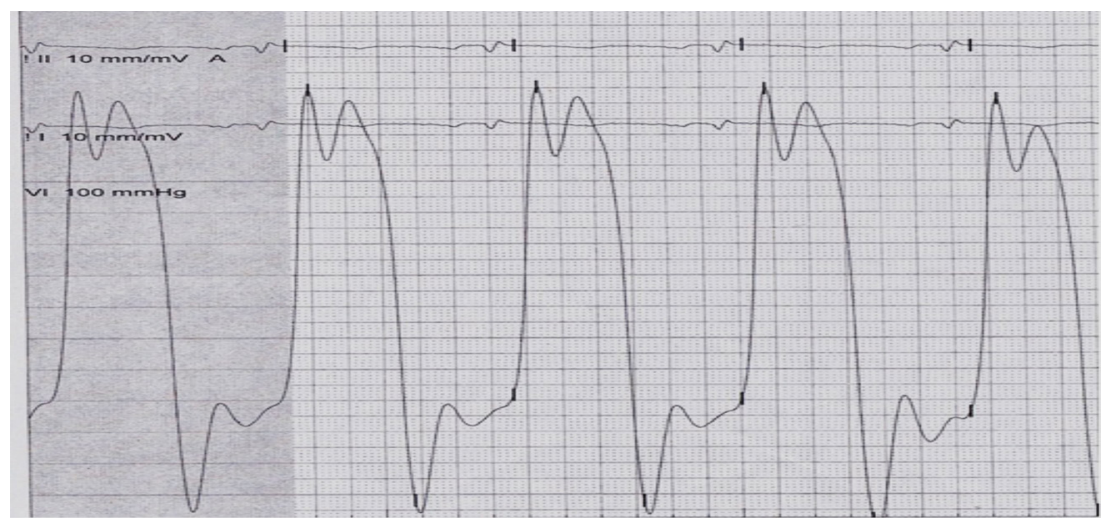

Figura 3. Curva hemodinámica. Curva de presión de ventrículo derecho con el signo de raíz cuadrada 
Con lo anterior se decide llevar a pericardiectomía total por esternotomía mediana con biopsia de pericardio, la cual reportó calcificación severa, sin presencia de granulomas ni bacilos acido alcohol resistente (BAAR) (figura 4), al igual que cultivo negativo para micobacterias.

Durante la cirugía se encontró pericardio severamente engrosado con calcificación en $70 \%$ de su superficie y diversas adherencias sujetas a la vena cava superior e inferior y aurícula derecha, llegando a haber perforación de la aurícula que se corrige con rafia, sin otras complicaciones. El paciente tuvo una notable mejoría de síntomas, teniendo un posoperatorio adecuado, logrando egreso cinco días después de la cirugía.
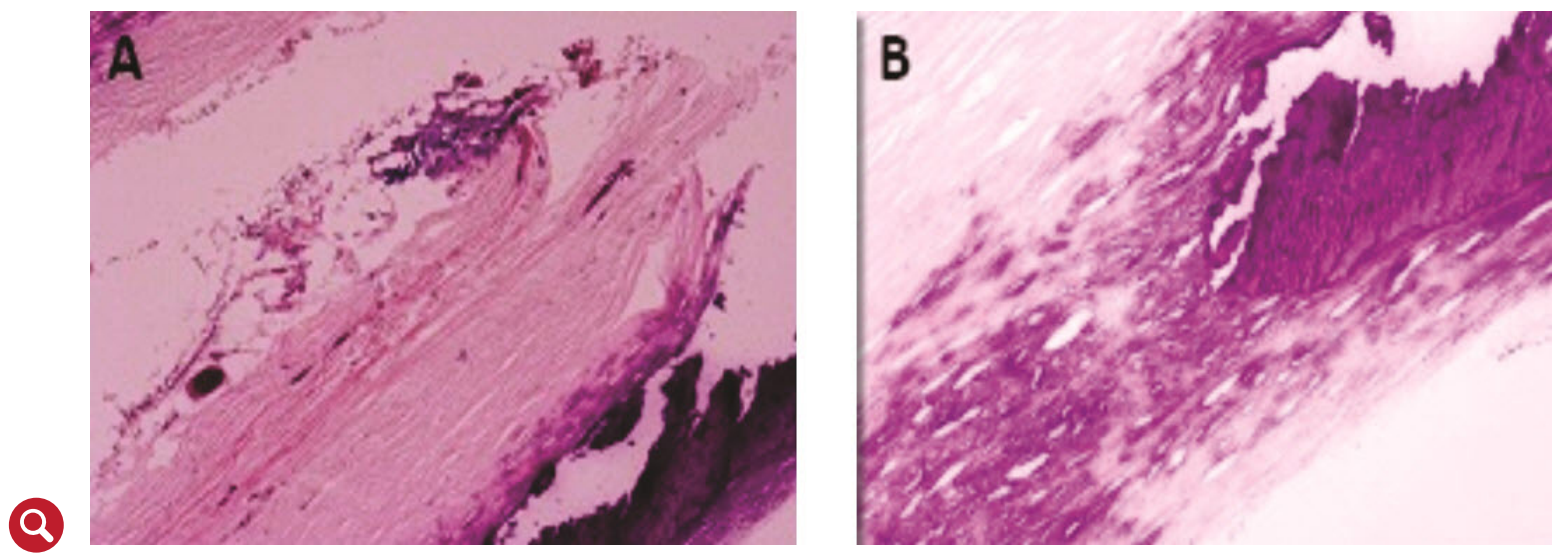

Figura 4. Biopsia de pericardio. (A) Se observa fibrosis de pericardio con engrosamiento difuso. (B) Calcificación pericárdica, Imagen de concretio cordis

En la gran mayoría de los pacientes con pericarditis constrictiva crónica no se puede establecer el diagnóstico etiológico, ni siquiera tras el estudio histológico del pericardio.

\section{Discusión}

En la pericarditis constrictiva crónica hay engrosamiento y fusión de ambas hojas del pericardio, aunque suele predominar la afección del pericardio parietal, que está muy rígido, inextensible y, en un 30 a 40 \% de los casos, calcificado (). El diagnóstico suele ser relativamente fácil, pero poco común y lo más importante para establecer este diagnóstico es tener un elevado índice de sospecha.

Entre las causas de pericarditis constrictiva están la idiopática y viral entre 42 - $49 \%$, post-cirugía cardíaca entre 11-37\%, post-radioterapia, principalmente después de la enfermedad de Hodgkin o cáncer de mama (9-31\%), las causadas por enfermedad del tejido conectivo (3 - $7 \%$ ), pericarditis tuberculosa o purulenta (3 - $6 \%$ ). Otras causas incluyen malignidad, trauma, fármacos, asbestosis, sarcoidosis, pericarditis urémica, con una prevalencia que oscila entre el 1 - $10 \%$ (4).

En la gran mayoría de los pacientes con pericarditis constrictiva crónica no se puede establecer el diagnóstico etiológico, ni siquiera tras el estudio histológico del pericardio. Cuanto mayor es la antigüedad de la pericarditis y mayor es el grado de calcificación del pericardio, menos probable es que el estudio histológico muestre datos que señalen una etiología específica. Por tanto, estos casos se califican de "idiopáticos". Es posible que algunos de estos enfermos hubieran sufrido una pericarditis viral o tuberculosa que pudo pasar inadvertida, pero ciertamente este antecedente no se recoge en la gran mayoría de los enfermos. 
La pericarditis constrictiva debe sospecharse ante todo enfermo que se presenta con un cuadro de falla cardiaca derecha y sin signos de falla cardiaca izquierda. Así pues, la ingurgitación yugular manifiesta es el signo clave encontrándose en el $93 \%$.
El seguimiento clínico prospectivo de los enfermos tras un primer brote de pericarditis aguda muestra que la evolución a pericarditis constrictiva crónica es muy rara después de una pericarditis viral (aproximadamente $1 \%$ de los casos), mientras que es bastante frecuente después de una pericarditis tuberculosa (40 - 50 \%) (ㄷ) . Siendo poco probable la segunda posibilidad en el caso descrito, ya que la prueba PPD fue negativa.

En la actualidad es rara la pericarditis por tuberculosis, siendo más frecuente en países en vía de desarrollo, donde ocurre en el 1 a $2 \%$ de los pacientes con tuberculosis y puede presentarse clínicamente de tres formas: derrame pericárdico (80\%), pericarditis constrictiva con derrame pericárdico (15\%) y pericarditis constrictiva (5\%) (6).

La pericarditis constrictiva debe sospecharse ante todo enfermo que se presenta con un cuadro de falla cardiaca derecha y sin signos de falla cardiaca izquierda. Así pues, la ingurgitación yugular manifiesta es el signo clave encontrándose en el $93 \%$ de los pacientes. La combinación de síntomas en casos de pericarditis constrictiva severa revela ascitis en el $37 \%$ de los pacientes, hepatomegalia (53\%), derrame pleural (35\%), edema periférico (76 \%); lo cual lleva a un falso diagnóstico de hepatopatía crónica. El signo de Kussmaul se presenta en el 21 \% de los pacientes y solo el $4 \%$ tienen frote pericárdico ().

La calcificación pericárdica se encuentra hasta en un 25 \% relacionada con una larga duración del proceso de constricción. Las radiografías de tórax que muestran un anillo de calcificación pericárdica sugieren pericarditis constrictiva en pacientes con signos y síntomas de falla cardíaca derecha. Esta calcificación se ve mejor en la proyección oblicua lateral.

En comparación con aquellos sin calcificación, los pacientes con calcificación tienen una mayor probabilidad de tener enfermedad pericárdica idiopática (67 \%), mayor duración de los síntomas, mayor probabilidad de tener frote pericárdico, aurículas de mayor tamaño, arritmias auriculares y mayor mortalidad perioperatoria (으)

La ecocardiografía es una herramienta diagnóstica que ayuda a diferenciar pericarditis constrictiva de cardiomiopatía restrictiva y debe ser la modalidad de imagen no invasiva inicial; Se describen tres signos básicos: 1. presencia de muesca septal que denota un cambio repentino en la posición del tabique ventricular, causada por una asimetría de llenado del ventrículo derecho y el ventrículo izquierdo y por lo tanto por los cambios rápidos en el diferencial de presión. 2. desplazamiento del septum interventricular con respiración, signo documentado en el caso clínico y, 3. aumento del tamaño biauricular: mientras mayor sea la ampliación es más compatible con cardiomiopatía restrictiva. Tiene una sensibilidad y especificidad diagnóstica del 85 y $90 \%$, respectivamente (9).

Los hallazgos en la tomografía computarizada de tórax incluyen aumento del grosor pericárdico > $4 \mathrm{~mm}$ y calcificación (el grosor normal del pericardio es de $2 \mathrm{~mm}$ ). Aunque se puede presentar pericarditis constrictiva con grosor pericárdico normal en el $18 \%$ de los pacientes (10).

Otros hallazgos en la tomografía computarizada que sugieren pericarditis constrictiva incluyen dilatación de la vena cava inferior, contornos ventriculares deformes, angulación del tabique ventricular. Un aspecto normal del pericardio no descarta la pericarditis constrictiva. La no visualización de la pared ventricular posterolateral 
Los diagnósticos diferenciales más comunes comprenden falla cardiaca aguda, tromboembolismo pulmonar, infarto del ventrículo derecho, derrame pleural, enfermedad pulmonar obstructiva crónica y cardiomiopatía restrictiva. izquierda en la tomografía computarizada dinámica puede indicar fibrosis del miocardio o atrofia y se asocia con un mal resultado quirúrgico (10).

Aunque la tomografía computarizada es superior a la imagen por resonancia magnética en la detección de calcificación, esta última es mejor para diferenciar pequeños derrames de engrosamiento pericárdico. También tiene el potencial de resolver los acontecimientos hemodinámicos como rebote septal e identificar mejor la inflamación del pericardio (11).

Es importante la evaluación hemodinámica para el diagnóstico de pericarditis constrictiva, aunque no siempre es necesaria. El hallazgo más importante es la equiparación de la meseta diastólica de la aurícula izquierda con la aurícula y el ventrículo derecho en un rango de $5 \mathrm{~mm} \mathrm{Hg} \mathrm{(12).}$

Talreja et al. reportan una sensibilidad de $97 \%$ y seguridad de predicción del $100 \%$ en pacientes con necesidad de procedimiento quirúrgico. El signo de la raíz cuadrada se observa durante el cateterismo derecho en pacientes con pericarditis constrictiva (13).

Los diagnósticos diferenciales más comunes comprenden falla cardiaca aguda, tromboembolismo pulmonar, infarto del ventrículo derecho, derrame pleural, enfermedad pulmonar obstructiva crónica y cardiomiopatía restrictiva.

La pericardiectomía es el tratamiento de elección en sitios idóneos para dicho procedimiento, siendo la mortalidad perioperatoria de 6 a $12 \%$. Como principal causa se describe el bajo gasto por falla cardíaca aguda, la cual es secundaria a atrofia y fibrosis miocárdica (14).

Las principales complicaciones incluyen falla cardiaca aguda perioperatoria y ruptura de la pared ventricular. La pericardiectomía parcial tiene 4,5 veces más riesgo de mortalidad comparado con pericardiectomía total, asociada a bajo gasto y constricción recurrente. En pacientes con clase funcional de NYHA estadio I o II se prefiere percardiectomía total

Los predictores de pobre pronóstico después de pericardiectomía en pacientes con pericarditis constrictiva crónica son la alta velocidad diastólica precoz del flujo mitral y la diabetes mellitus, lo que indica que la valoración mediante ecocardiografía no solo puede ser útil en el diagnóstico sino que predice pronóstico después de la pericardiectomía (15).

\section{Conclusión}

La calcificación pericárdica es un hallazgo poco frecuente que debe ser tenido en cuenta posterior a un episodio de pericarditis aguda. Aunque en la mayoría de las series su etiología es idiopática, posiblemente asociado al tiempo de evolución, no se debe olvidar que en nuestro medio la tuberculosis cuenta con un gran porcentaje de los casos y en ocasiones solo se llega a este diagnóstico mediante estudio histopatológico o cultivo de la muestra. Un adecuado abordaje diagnóstico basado en la clínica y hallazgos imagenológicos son la piedra angular para una exitosa aproximación terapéutica.

\section{Conflictos de interés}

Este trabajo fue financiado con fondos propios, los autores manifiestan no tener ningún conflicto de interés. 


\section{Bibliografía}

1. Bertog SC, Thambidorai SK, Parakh K, Schoenhagen P, Ozduran V, Houghtaling $\mathrm{PL}$, et al. Constrictive pericarditis: etiology and cause-specific survival after pericardiectomy. J Am Coll Cardiol. 2004 Apr 21;43(8):1445-52. https://www.ncbi. nlm.nih.gov/pubmed/15093882

2. Isner JM, Carter BL, Bankoff MS, Konstam MA, Salem DN. Computed tomography in the diagnosis of pericardial heart disease. Ann Intern Med. 1982 Oct;97(4):4739. https://www.ncbi.nlm.nih.gov/pubmed/7125406

3. Sagristà-Sauleda J. Cardiac constriction syndromes. Rev española Cardiol. 2008 Jun;61 Suppl 2:33-40. https://www.ncbi.nlm.nih.gov/pubmed/18590635

4. Imazio M, Brucato A, Maestroni S, Cumetti D, Belli R, Trinchero R, et al. Risk of constrictive pericarditis after acute pericarditis. Circulation. 2011 Sep 13;124(11):1270-5. https://www.ncbi.nlm.nih.gov/pubmed/21844077

5. Sagristà-Sauleda J, Permanyer-Miralda G, Soler-Soler J. Tuberculous pericarditis: ten year experience with a prospective protocol for diagnosis and treatment. J Am Coll Cardiol. 1988 Apr:11(4):724-8. https://www.ncbi.nlm.nih.gov/ pubmed/3351140

6. Mayosi BM, Wiysonge CS, Ntsekhe M, Volmink JA, Gumedze F, Maartens G, et al. Clinical characteristics and initial management of patients with tuberculous pericarditis in the HIV era: the Investigation of the Management of Pericarditis in Africa (IMPI Africa) registry. BMC Infect Dis. 2006;6:2. http://bmcinfectdis.biomedcentral.com/articles/10.1186/1471-2334-6-2

7. Ling LH, Oh JK, Schaff H V, Danielson GK, Mahoney DW, Seward JB, et al. Constrictive pericarditis in the modern era: evolving clinical spectrum and impact on outcome after pericardiectomy. Circulation. 1999 Sep 28;100(13):1380-6. https:// www.ncbi.nlm.nih.gov/pubmed/10500037

8. Talreja DR, Edwards WD, Danielson GK, Schaff H V, Tajik AJ, Tazelaar HD, et al. Constrictive pericarditis in 26 patients with histologically normal pericardial thickness. Circulation. 2003 Oct 14;108(15):1852-7. http://circ.ahajournals.org/ content/108/15/1852

9. Himelman RB, Lee E, Schiller NB. Septal bounce, vena cava plethora, and pericardial adhesion: informative two-dimensional echocardiographic signs in the diagnosis of pericardial constriction. J Am Soc Echocardiogr. 1988 SepOct;1(5):333-40 https://www.ncbi.nlm.nih.gov/pubmed/3272782

10. Verhaert D, Gabriel RS, Johnston D, Lytle BW, Desai MY, Klein AL. The role of multimodality imaging in the management of pericardial disease. Circ Cardiovasc Imaging. 2010 May;3(3):333-43. https://www.ncbi.nlm.nih.gov/pub$\underline{\mathrm{med} / 20484113}$

11. Sechtem U, Tscholakoff D, Higgins CB. MRI of the normal pericardium. AJR Am J Roentgenol. 1986 Aug;147(2):239-44. https://www.ncbi.nlm.nih.gov/pub$\underline{\text { med/3487935 }}$ 
12. Sorajja P. Invasive hemodynamics of constrictive pericarditis, restrictive cardiomyopathy, and cardiac tamponade. Cardiol Clin. 2011 May;29(2):191-9. https://www.ncbi.nlm.nih.gov/pubmed/21459242

13. Talreja DR, Nishimura RA, Oh JK, Holmes DR. Constrictive pericarditis in the modern era: novel criteria for diagnosis in the cardiac catheterization laboratory. J Am Coll Cardiol. 2008 Jan 22;51(3):315-9. http://content.onlinejacc.org/article. aspx?articleid $=1187170$

14. Schwefer M, Aschenbach R, Heidemann J, Mey C, Lapp H. Constrictive pericarditis, still a diagnostic challenge: comprehensive review of clinical management. Eur J Cardiothorac Surg. 2009 Sep;36(3):502-10. https://www.ncbi.nlm.nih.gov/ pubmed/19394850

15. Kang SH, Song J-M, Kim M, Choo SJ, Chung CH, Kang D-H, et al. Prognostic predictors in pericardiectomy for chronic constrictive pericarditis. J Thorac Cardiovasc Surg. 2014 Feb;147(2):598-605. https://www.ncbi.nlm.nih.gov/pubmed/23380514 